\title{
Protective Effects of a Novel Preparation Consists of Concentrated Dead Sea Water and Natural Plants Extracts against Skin Photo-Damage
}

\author{
Meital Portugal-Cohen ${ }^{1 *}$, Isabelle Afriat-Staloff ${ }^{1}$, Yoram Soroka ${ }^{2}$, Marina Frusic-Zlotkin ${ }^{2}$, \\ Gerrit Schlippe ${ }^{3}$, Werner Voss ${ }^{3}$, Ze'evi Ma'or ${ }^{1}$
}

${ }^{1}$ AHAVA-Dead Sea Laboratories, Lod, Israel; ${ }^{2}$ School of Pharmacy, Institute for Drug Research, Faculty of Medicine, The Hebrew University of Jerusalem, Jerusalem, Israel; ${ }^{3}$ Dermatest ${ }^{\circledR}$ Medical Research Company, Muenster, Germany.

Email: *meital.p@ahava.com, isabelle.a@ahava.com, yorams@ekmd.huji.ac.il, marina.zlutkinfrusic@mail.huji.ac.il, dr.shlippe@dermatest.de, dr.voss@dermatest.de, zeevi.m@ahava.com

Received November 21 $1^{\text {st }}$, 2013; revised December $20^{\text {th }}$, 2013; accepted December $28^{\text {th }}, 2013$

Copyright (C) 2014 Meital Portugal-Cohen et al. This is an open access article distributed under the Creative Commons Attribution License, which permits unrestricted use, distribution, and reproduction in any medium, provided the original work is properly cited. In accordance of the Creative Commons Attribution License all Copyrights (C) 2014 are reserved for SCIRP and the owner of the intellectual property Meital Portugal-Cohen et al. All Copyright (c) 2014 are guarded by law and by SCIRP as a guardian.

\section{ABSTRACT}

Background: Exposure to solar UV rays has deleterious effects on skin appearance through physiological and structural alterations that eventually lead to skin photo-damage. Aims: To test the photo-protective effect of a novel preparation, Dead Sea Osmoter Concentrate (DSOC), containing high concentration of Dead Sea water. Methods: Human skin organ culture was used as a model to assess the biological effects of UVB irradiation and the protective effect of topical application of DSOC preparation. Skin pieces were analyzed for mitochondrial activity by Alamar blue (resazurin) assay, for apoptosis by caspase 3 assay, for detoxification by proteasome $20 \mathrm{~S}$ sub-unit activity and for skin hydration by aquaporin 3 (AQP3) membrane channels expression. Human subjects were tested to evaluate the effect of DSOC Serum, a topical formula for facial skin, on skin hydration by a corneometer. Results: UVB irradiation induced cell apoptosis in the epidermis of skin organ cultures and decreased their proteasome activity and AQP3 expression. Topical application of DSOC significantly attenuated all these effects. In human subjects, an elevation in the intense skin moisture, which was higher relatively to subjects' regular moisturizer, was observed. Moreover, DSOC Serum boosted the hydration effect together with regular moisturizer. Conclusions: The results clearly demonstrate that DSOC preparation can significantly improve the skin capability in coping with UVB rays stress in different cellular functions such as anti-apoptotic properties as well as detoxification and hydration recovery that can attenuate biological effects of skin photo-damage. Topical application of DSOC Serum had contributed to skin appearance by a strong hydration impact, also as a booster.

\section{KEYWORDS}

Photo-Damage; Skin Dryness; Skin Barrier Function; Skin Detoxification; Dead Sea Minerals

\section{Introduction}

Skin, as the outermost organis exposed extensively to UV radiation-derived from sunlight, that can stimulate photochemical and photo-biological reactions, which eventually leads to tissue damage and could cause skin cancer $[1,2]$. Chronic exposure to UV radiation is involved with photo-damage and accelerates skin aging

${ }^{*}$ Corresponding author. characterized by dry and rough surface, reduction of elasticity, wrinkles' formation and hyper-pigmentation [3]. Skin exposure to UVB rays (280 - $320 \mathrm{~nm})$ is related to photo-damage associated mainly with epidermis layers but it can also affect the dermal layer [4]. Since UVB photo-damage is an important component of UV damage, a protection against UVB photo-damage is very important from both skin care and dermatology points of view.

Epidermis is one of the paramount target organs to en- 
vironmental oxidative damage due to its location, as our body outermost layer, where it is always in contact with oxygenic air and occasionally exposed to UVB (mainly solar) light. Over-production of reactive oxygen species (ROS) could result in structural and functional alterations of cutaneous proteins resulted in loss of enzymatic activity, altered cellular functions such as energy production, interference with the creation of membrane potentials, and changes in the type and level of cellular proteins. Protein oxidation products are usually aldehydes, keto compounds, and carbonyls [5].

Additional major characteristic of photo-damaged skinis its dehydration status. Water is essential for vital skin functioning and particularly its outer layer, the stratum corneum (SC). Environmental daily insults, including sun exposure, hot dry air, and winds might lower the SC water content, causing improper desquamation and the appearance of dry, flaky skin [6]. Aquaporin 3 (AQP3), a member of the family of aquaglyceroporins, is a small, hydrophobic, integral membrane protein that act primarily as water-selective pores, thus contributes to balance the water level and to water loss prevention [7]. AQP3 provides a short circuit for water between the base of the epidermis and the SC, maintains constant water content. [6] and it is known to be down regulated following UVB irradiation [8].

Face is one of the most UVB exposed area in our body and thus susceptible to pre-mature skin aging. Sunscreen application supplies only limited defense against the long-term consequences of UV exposure, therefore, additional treatment strategies should be considered for enhance skin protection. Various natural components can contribute to non-sunscreen solar protection and their activity can assist in skin protection by intervention in metabolic pathways involved in UV damages.

Dead Sea (DS) water, salts, minerals and mud are well known for their therapeutic activities and are used in treating a variety of skin conditions as well as for their cosmetic benefits $[9,10]$. Osmoter ${ }^{\mathrm{TM}}$, a natural commercial composition of DS water was shown to reduce skin senescence markers, to attenuate UVB damage markers [11] and to improve skin smoothness [12]. Inclusion of extracts isolated directly from plants and other natural sources for therapeutic and cosmetic purposed can contribute and improve solar protection.

Dead Sea Osmoter Concentrate (DSOC) preparation (US patent application pending 61/812,946), formulated by AHAVA Dead Sea Laboratories LTD, is a unique combination of natural actives consisting of concentrated Dead Sea Minerals extract (Osmoter ${ }^{\mathrm{TM}}$ ), at a relatively high concentration, with Green tea extract, Grape seed extract, Olive leaf extract and Benzotriazolyl Dodecyl p-Cresol in water. These extracts were selected based on bio-activity measured in a screening process of a list of candidate actives we had performed ex vivo on human skin organ cultures with and without exposure to UVB irradiation (data is not shown).

In this study, the capabilities of DSOC to protect human skin against UVB-induced biological damaging impacts were investigated on $e x$-vivo human skin model and in-vivo clinically on human volunteers.

\section{Methods}

\subsection{Treatment Preparations}

All preparations used in this study were manufactured by AHAVA Dead Sea Laboratories, Ltd., Israel.

\subsection{Dead Sea Osmoter Concentrate ${ }^{\mathrm{TM}}$ Preparation}

Dead Sea Osmoter Concentrate ${ }^{\mathrm{TM}}$ (DSOC) preparation: Osmoter ${ }^{\mathrm{TM}}$ solution, Benzotriazolyl Dodecyl p-Cresol (Tinogard TL), green tea extract (Greenselect), grape seed extract (Leucoselect) and olive leaf extract (Eurol BT) in water.

Osmoter $^{\mathrm{TM}}$ : The major constitutes of DS water are the following ions: $\mathrm{Mg}^{2+}(92,500 \mathrm{mg} / \mathrm{l}), \mathrm{Ca}^{2+}(38,000 \mathrm{mg} / \mathrm{l})$, $\mathrm{K}^{+}(1400 \mathrm{mg} / \mathrm{l}), \mathrm{Na}^{+}(2000 \mathrm{mg} / \mathrm{ml}), \mathrm{Sr}^{2+}$ (800 mg/l), $\mathrm{Cl}^{-}$ (345,000 mg/l), $\mathrm{Br}^{-}(11,500 \mathrm{mg} / \mathrm{l})$.

\subsection{DSOC Serum Preparation}

DSOC preparation was further incorporated into topical preparation in a form of a serum (DSOC Serum). The various additional ingredients comprised within the DSOC Serum are as follows: Aqua (Mineral Spring Water), PVP, Maris Aqua (Dead Sea Water), Cyclomethicone, Dimethicone, Butylene Glycol \& Sigesbeckia Orientalis Extract \& Rabdosia Rubescens Extract \& Aqua (Water), Caprylyl Methicone \& PEG-12 Dimethicone/ PPG-20 Crosspolymer, Aloe Barbadensis Leaf Juice, Glycerin, Isodecyl Isononanoate, Diisobutyl Adipate, Dimethicone \& Dimethiconol, Phenoxyethanol \& Aqua (Water) \& Biosaccharide Gum-1, Sodium Lactate, Trehalose, Butylene Glycol \& Phenoxyethanol \& Laminaria Digitata Extract, Citric Acid \& Glycerin \& Lactic Acid \& Parfum (Fragrance) \& Phenoxyethanol \& Aqua (Water) \& Ascorbic Acid \& Citrus Reticulata Fruit Extract \& Citrus Aurantium Amara Fruit Extract, Bisabolol, Squalene (Phytosqualene), Argania Spinosa Kernel Oil, Parfum (Fragrance), Hydroxyethyl AcrylatelSodium Acryloydimethyl, Sodium Hyaluronate, Benzotriazolyl Dodecyl pCresol, Helianthus Annuus (Sunflower) Seed Oil \& Aqua (Water) \& Ascorbic Acid \& Melissa Officinalis Leaf Extract \& Polyglyceryl-3 polyricinoleate, Tris(tetra-methyl hydroxyl piperidinol) citrate, Sodium Benzotriazolyl 
Butylphenol Sulfonate, Glucosyl Hesperidin, Aqua (Water) \& Olea Europaea (Olive) Leaf Extract \& Fructose, Pentaerythrityl Tetra-di-t-butyl Hydroxyhydrocin, Vitis Vinifera (Grape) Seed Extract \& Phosphatidylcholine, Camellia Sinensis (Green Tea) Leaf Extract \& Phosphatidylcholine.

\subsection{Human Skin Organ Culture Model for Biological Tests}

Skin fragments were obtained with informed consent from 20 - 60 years old, healthy women, who underwent breast or abdomen reduction. Samples were cut into pieces of $5 \times 5 \mathrm{~mm}$. Each experiment consisted of 4 replications for each treatment. The examined preparations were applied as a thin layer on the epidermis of each skin piece $(2.5 \mu \mathrm{l} / \mathrm{piece})$. The skin pieces were incubated in 12-wells culture plates filled with DMEM culture media (Dulbecco's Modified Eagle's Medium, Biological Industries BeitHaemek, Israel); $\left(37^{\circ} \mathrm{C} ; 5 \% \mathrm{CO}_{2}\right)$ so that the epidermis was exposed to the air while the dermis was submerged in the culture media. After $24 \mathrm{hr}$ of incubation, the preparations were wiped off from the skin on a sterile filter paper, the skin was washed in PBS and irradiated by a UVB lamp (VL-6.Mlamp, emission spectrum 280 - $350 \mathrm{~nm}$, emission peak $312 \mathrm{~nm}$, filter size $145 \times 48$ $\mathrm{mm}$, Vilber Lourmat, Torcy, France.). The irradiation intensity was $2 \mathrm{~mJ} / \mathrm{cm}^{2} / \mathrm{sec}$. Irradiation time was calculated in order to supply $250 \mathrm{~mJ} / \mathrm{cm}^{2}$. Freshly preparations were applied on the skin pieces and they were further incubated in culture plates containing replenished medium.

At the end of the post-irradiation period (which depended on the examined parameter), the skin was incubated for $1 \mathrm{~min}$ in phosphate buffered saline (PBS) at $56^{\circ} \mathrm{C}$ and the epidermis was separated from the dermis with a scalpel. The remaining culture medium was collected, frozen and kept at $-70^{\circ} \mathrm{C}$ until use.

\subsection{Apoptosis Determination by Caspase 3 Activity Assay}

Apoptosis determination by caspase 3 assay was carried out $24 \mathrm{hr}$ following UVB irradiation. For Caspase-3 activity measurement, the collected epidermis pieces were placed into wells of a 96-well plate, each filled by $125 \mu \mathrm{l}$ of Caspase-3 specific substrate solution $(10 \mu \mathrm{M} \mathrm{Ac-}$ DEVD-AMC, Merck, Darmstadt, Germany) with 0.02\% Triton X-100 (J. T. Baker, Phillipsburg, NJ, USA) and 10 mM DTT (TCI, Tokio, Japan) (34). The enzyme's fluorescent product emission level $(355 / 460 \mathrm{~nm})$ was measured at $37^{\circ} \mathrm{C}$ at 2-minute intervals using fluorescence plate reader Fluostar-BMG spectrofluorimeter (Offenburg, Germany) for $40 \mathrm{~min}$. Apoptosis level was later deduced from the slope value of enzymatic activity in the linear range.

Osmoter ${ }^{\mathrm{TM}}$ solutions in water at a concentration range of $0.8 \%$ and $4 \%$ were tested on ex vivo human skin organ cultures in order to determine the relevant Osmoter ${ }^{\mathrm{TM}}$ concentration in DSOC.

\subsection{Viability Measurements by Mitochondrial Assay}

Skin viability was determined using Alamar blue test using resazurin reagent, which is a redox indicator that both fluoresces and changes color in response to chemical reduction of the growth medium resulting from cell growth. Fresh growth medium containing $0.01 \mathrm{mg} / \mathrm{ml}$ resazurin (Sigma Aldrich, Israel) was added to cultures at the end of the test period and incubated for 2 hrs. Fluorescence at $540 \mathrm{~nm}$ (excitation)/590 nm (emission) proportional to mitochondrial activity was measured on a BioTek Synergy 2 microplate reader.

\subsection{Proteasome Activity Determination}

Proteasome activity of 20S sub-unit was measured following DSOC preparation application $72 \mathrm{hr}$ post irradiation on epidermis samples as adapted for Bregere et al. [13]. For testing its chymotrypsin-like-and trypsin-like activity, epidermis samples were peeled and incubated in 125 ul PBS containing LLVY-AMC as a substrate, with $0.02 \%$ Triton $\mathrm{X}-100$ and $10 \mathrm{~mm} \mathrm{DTT}$, at $37^{\circ} \mathrm{C}$ in a $96-$ well plate Fluorescence of the released coumarin derivative was measured at 360/460 nm, using a Fluostar-BMG spectrofluorimeter. Activity was measured by the fluorescence-versus-time slope, calculated over $30 \mathrm{~min}$ in the linear range.

\subsection{Aquaporin 3 (AQP3) Levels Determination}

The degree of Aquaporin protein expression in epidermis was determined following DSOC preparation application $48 \mathrm{hr}$ post irradiation. Prior to that, AQP3 expression was tested on epidermis $48 \mathrm{hr}$ following application of Osmoter ${ }^{\mathrm{TM}}$ in different concentrations without irradiation. AQP3 levels were determined by dot-blot analysis. Each separated epidermis sheet was placed in $500 \mu \mathrm{l}$ of sample buffer (Tris $0.25 \mathrm{M} \mathrm{pH}$ 6.8, SDS 1\%, Glycerol 5\%, $\beta$-mercaptoethanol 5\%), boiled for 5 minutes and kept at $20^{\circ} \mathrm{C}$ until use. Protein concentration in boiled samples was determined. Briefly, $3 \mu \mathrm{l}$ of each sample were spotted onto Whatman paper $3 \mathrm{MM}$, air-dried, rinsed with methanol and stained with Coommassie stain. After comparison to BSA standard curve, all the samples were diluted to $0.33 \mathrm{~g} / \mathrm{ml}$ in sample buffer. For immuno analysis, $3 \mu \mathrm{l}$ of thew samples ( $1 \mu \mathrm{g}$ of protein), in 4 replicates were deposited directly onto nitrocellulose membrane 
(dot blots). The membrane was processed as using antigen-specific primary antibody (ab15117, Abcam Cambridge, UK) and horseradish peroxidase-conjugated, antirabbit secondary antibody (Jackson Immuno Research, West Grove, PA, USA). Chemoluminescence was detected using a substrate cocktail composed of: $1.25 \mathrm{mM}$ luminol, $0.2 \mathrm{M}$ p-coumaric acid and $0.009 \% \mathrm{H}_{2} \mathrm{O}_{2}$ in 100 $\mathrm{mM}$ Tris/HCl pH 8.8, in a Fujifilm LAS 3000 instrument (Fujifilm, Tokyo, Japan). Densitometric analysis was performed using the NIH Image J program.

\subsection{Clinical Measurements for Skin Hydration}

Measurements of skin hydration were performed by Dermatest ${ }^{\circledR} \mathrm{GmbH}$ medical research clinic, in Munster, Germany. The study was carried out on 35 female subjects aged $18-74$ years on the forearm.

No patient was enrolled without his or her signed and witnessed informed consent. The study was approved by the Institutional and Ministry of Health Ethics Committee.

The measurements were performed over a defined period of time-(0.5-hr, 8-hr, 24-hr), after a single application to 4 different application areas in the flexor aspects of the forearms. In the first area only the DSOC Serum was applied, in the second area only the Regular Moisturizer of the subjects, in the third area the DSOC Serum plus the Regular Moisturizer. The last area served as control area, nothing was applied.

The subjects were instructed to apply only the cosmetic preparation being tested on the relevant pre-determined skin areas during the application period. The test subjects were acclimatized for 45 minutes at a temperature of $22^{\circ} \mathrm{C}$ and $60 \%$ relative humidity. Skin values were measured at three different places within the respective testing areas. Electrical capacitance was measured with a capacitance meter (Corneometer CM 825, Courage \& Khazaka, CK). The probe head consisting of a condenser was applied on the skin surface at constant pressure. The range values of each measurement were recorded and compared to the other measurements.

The recorded values were averaged. For this experiment, the untreated skin close to the test area was used as the control measurement area. Measurements were taken before the application and 30 minutes, 8 hours and 24 hours after a single application.

The results represent the average percentage moisture change caused by the application with reference to starting value.

Inclusion criteria: subjects with a lack of skin tone homogeneity/slight discolorations and with dehydrated skin in the test area aged 18 years and older.

Exclusion criteria: severe or chronic skin inflammation; serious inner or chronic diseases; intake of drugs that possibly can interfere with skin reactions (Glucocorticoids, antiallergics, topical immuno modulator, etc.); application of pharmaceutical products and skin care products with active ingredients until 7 - 10 days before testing; severe allergies or occurred severe side effects after usage of cosmetic products; sunbath or usage of tanning bed during the study period; known cancer; pregnancy or lactation period.

\subsection{Adverse Events and Tolerance}

Before the start of the application test all participants were determined to have healthy skin in the test area. No pathological skin disorder was detected. During the study: No complaint of any pathological skin disorder was reported during the course of this application test. Interruptions of the application test and/or medical intervention were not necessary.

After the end of the application test: During the final dermatological examination after the end of the study, none of the 35 participants showed development of any pathological skin disorder in the test area. The mentioned product was well-tolerated and did not lead to any unwanted skin reaction.

\subsection{Data Analysis}

Average values are given with standard error of the mean (SEM). Differences between average values were tested for significance using the Two-tailed Student t-test for ex vivo experiments and Dunnett test for in vivo experiments and considered significant for $\mathrm{p} \leq 0.05$.

Each ex vivo experiment was performed at least in triplicate.

\section{Results}

\subsection{Ex-Vivo Results}

The efficacy of topical application of DSOC preparation: Skin specimens were applied with DSOC preparation and UVB-irradiated. Skin biomarkers for apoptosis, viability, detoxification and hydration were measured in skin specimens with and without UVB irradiation stress.

The significant enhancement in pro-apoptotic caspase 3 enzyme activity-induced by UVB irradiation, was markedly decreased when skin was pre-treated topically with DSOC preparation: 34\% compared with non-treated samples ( $<$ < 0.05) (Figure 1). The application of DSOC preparation did not induce apoptosis in non-irradiated samples.

Alamar blue (resazurin) mitochondrial activity assay was used for analyzing skin viability. Topical application of DSOC preparation did not impede mitochondrial activity in both non-irradiated and irradiated samples. Moreover, pretreatment by DSOC preparation enhanced 
mitochondrial activity in irradiated samples by $75 \%$ ( $<<$ 0.01) (Figure 2).

The proteasome is the major intracellular proteolytic system implicated in the deletion and recycle abnormal and oxidized proteins. In human epidermal cells, previous studies have evidenced that proteasome function is decreased during aging as well as upon UV irradiation. Proteasome activity was used to indicate the rate of degradation of unwanted or damaged proteins in the epidermis. Results showed that UVB irradiation in untreated samples led to a significant decrease of $40 \%(\mathrm{p}<0.05)$ in 20 S sub-unit activity (Figure 3). Pre-treatment with DSOC preparation prevented this decrease.

AQP3, a membranal protein acting as water/glycerol transporter, is an important contributor for skin hydration.

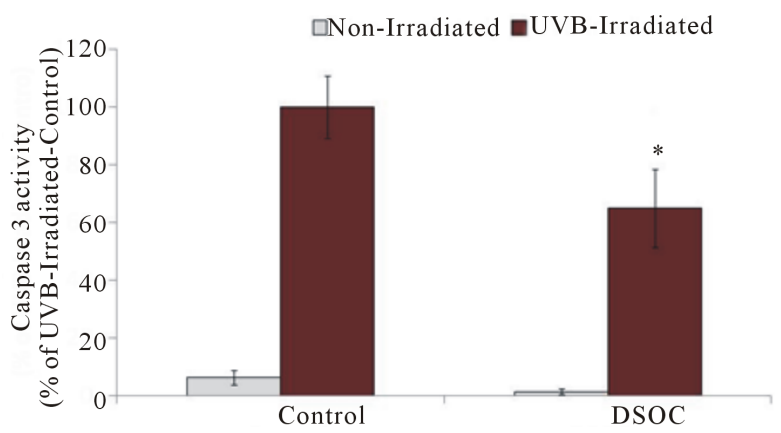

Figure 1. Epidermal apoptosis following treatment with DSOC preparation and UVB irradiation. Human skin pieces were applied with CO preparation for $24 \mathrm{hrs}$ and UVB irradiated at $250 \mathrm{~mJ} / \mathrm{cm}^{2}$ after the removal of preparation. Following irradiation, DSOC preparation was applied again and the skin pieces were further incubation. Caspase 3 activity was measured in epidermal extracts after 24 hrs following irradiation Data are presented as Mean \pm SEM. ${ }^{*} \mathbf{p}<$ 0.05 Irradiated-DSOC applied vs. Irradiated Control.

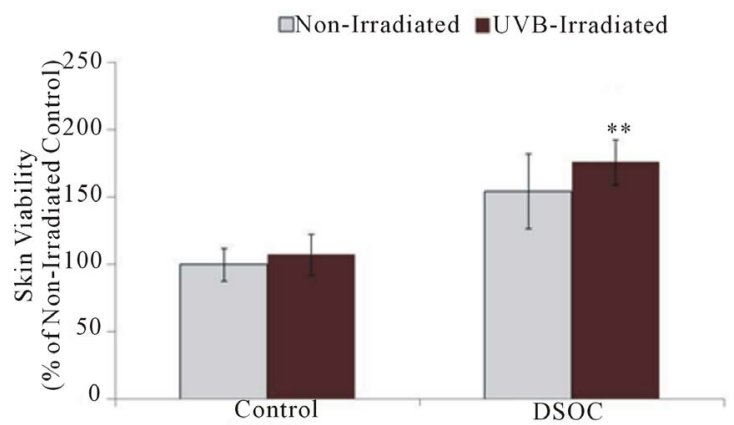

Figure 2. Skin viability following treatment with DSOC preparation and UVB irradiation. Human skin pieces were applied with DSOC preparation for 24 hrs and UVB irradiated at $250 \mathrm{~mJ} / \mathrm{cm}^{2}$ after the removal of preparation. Following irradiation, DSOC preparation was applied again and the skin pieces were further incubation. Cell viability was evaluated by Alamar blue (resazurin) assay 72 hrs following irradiation. Data are presented as Mean \pm SEM. ${ }^{* *} \mathrm{p}$ $<0.01$ Irradiated-applied vs. Irradiated Control.

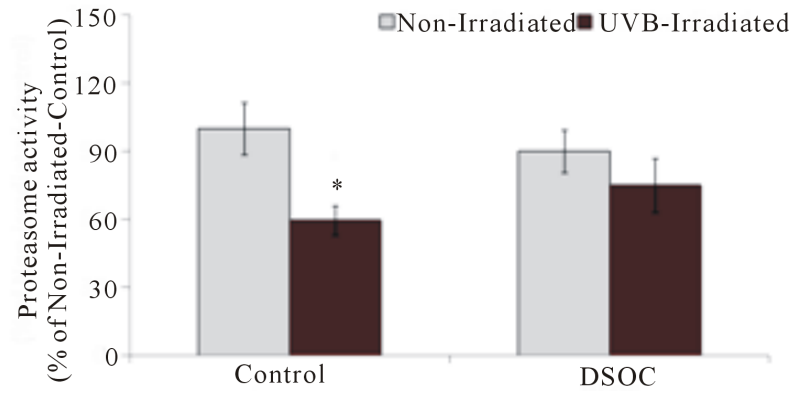

Figure 3. Epidermal proteasome activity following treatment with DSOC preparation and UVB irradiation. Human skin pieces were applied with DSOC preparation for 24 hrs and UVB irradiated at $250 \mathrm{~mJ} / \mathrm{cm}^{2}$ after the removal of preparation. Proteasome activity was evaluated using fluorescence measurement with LLVY-AMC as a substrate in epidermal extracts 72 hrs following irradiation. Data are presented as Mean \pm SEM. ${ }^{*}$ p $<0.05$ Irradiated-DSOC applied vs. Irradiated Control.

UVB irradiation decreased AQP3 protein levels in epidermis of untreated samples by $17 \%(\mathrm{p}<0.05)$ (Figure 4). Pre-treatment with DSOC preparation markedly abolished this decrease: AQP3 level in irradiated treated samples was $88 \%$ higher than in irradiated untreated ones $(\mathrm{p}<0.05)$. Also, an increase in AQP3 expression by $44 \%$ $(\mathrm{p}=0.05)$ was observed in non-irradiated skin pretreated with DSOC compared to non-irradiated control.

\subsection{In-Vivo Clinical Results}

Skin hydration was evaluated before and after topical application of the following treatments: DSOC Serum, Regular Moisturizer and DSOC Serum together with topical application of regular moisturizer. A significant elevation in skin hydration was presented $0.5 \mathrm{hr}$ and $8 \mathrm{hr}$ after all treatments application compared to the control area: $105 \%$ for DSOC Serum (p < 0.01), 90\% for Regular Moisturizer $(p<0.01)$ and $136 \%$ for DSOC Serum + Regular Moisturizer ( $<$ < 0.01) after $0.5 \mathrm{hr} ; 50 \%$ for DSOC Serum ( $\mathrm{p}<0.01$ ), 41\% for Regular Moisturizer ( $\mathrm{p}$ $<0.01$ ) and $51 \%$ for DSOC Serum + Regular Moisturizer $(\mathrm{P}<0.01)$ after 8 hrs. (Figure 5). $24 \mathrm{hr}$ after treatments application, a significant elevation in skin hydration was observed only after DSOC Serum and DSOC Serum+ Regular Moisturizer application compared to control area: $17 \%$ for DSOC Serum $(\mathrm{p}<0.01)$ and $21 \%$ for DSOC Serum + Regular Moisturizer $(\mathrm{p}<0.01)$.

\section{Discussion}

Skin exposure to Ultraviolet (UV) radiation plays an important role in the pathogenesis of skin aging and skin cancers. Epidermis, the outermost skin layer, is primarily affected by UV Brays, which can lead to photo-damaged phenotype due to its involvement in molecular oxidation, 
inflammation and dryness [14]. Hence, a topical application of protecting preparations, possessing biological activities, such as recycling damaged oxidized substances (detoxification), anti-inflammatory properties and at the same time maintaining skin moisture, may be able to attenuate the process of skin photo-damage.

This study is focused on a Dead Sea Osmoter ${ }^{\mathrm{TM}}$ Concentrate (DSOC), a novel preparation consisting of Osmoter ${ }^{\mathrm{TM}}$ - $\mathrm{a}$ special mixture of DS water extract highly concentrated, Green tea extract, Grape seed extract, Olive leaf extract and Benzotriazolyl Dodecyl p-Cresol in water.

The therapeutic properties of DS water are well established and are related to the unique mineral composition, mainly dissolved salts of magnesium, calcium, sodium, potassium, zinc and strontium. Many DS based compositions have been used to also alleviate skin disorders symptoms. Studies have investigated the role and mechanisms of the DS minerals on skin [11]. Some studies have focused on isolating the effect of particular minerals and some of the semetallic elements from DS water have been proven to be involved in skin metabolism: zinc ions were reported to enhance epidermal proliferation and wound healing [15]. Magnesium and calcium ions are known as skin barrier recovery accelerators. DS water has been reported to stimulate cellular proliferation and mitochondrial activity, to decrease the expression of aging bio-markers and to diminish apoptotic damage after skin exposure to UVB irradiation [11]. In this study, we further examine the effect of DS minerals in association with plant extracts on essential skin functions and with regards to UVB irradiation, a main component of skin aging.

Green tea (Camellia Sinensis) leaf extract is rich in poly-phenols. The most abundant and biologically active poly-phenol is the catechinepigallocatechin-3-gallate (EGCG), which is a potent antioxidant. Many studies have shown that topical treatments or oral consumption of green tea or its poly-phenols can inhibit skin tumorigenesis, induced bycemical carcinogens or ultraviolet radiation. Recent Study has shown that Green tea leaf extract also possesses anti-inflammatory activity [16].

Grape (Vitis Vinifera) seed extract is rich in pro-anthocyanidins, potent antioxidants reported topossess more free radical scavenging activities than vitamins $\mathrm{C}$ and $\mathrm{E}$ [17]. Grape seed extract is also known to protect skin against UVB irradiation through inhibiting the depletion of antioxidant defense components induced by UVB [18].

Olive (Olea Europaea) leaves have long been used in folk medicine in Mediterranean countries. Oleuropein, the main polyphenolic compound in Olive leaf extract, and its derivatives have tested for a variety of biochemi- cal activities. Olive leaf extract, and its derivatives were reported to effective. It prevents LDL oxidation [19], attenuate chronic UVB-induced skin damage, carcinogenesis and tumor growth, probably due to inhibition of the expression of vascular endothelial growth factor (VEGF) and Metalloproteinases (MMP-2, MMP-9, MMP-13), through a reduction in COX-2 levels [20].

Benzotriazolyl Dodecyl p-Cresol (commercially available as Tinogard TL) is formulated to stabilize transparent skincare preparations. It protects sensitive ingredients such as colorants, fragrances, and natural extracts from photolytic and/or photo-oxidative degeneration, thus providing long-lasting shelf life (BASF, The Chemical Company).

In this study, the biological effects of DSOC were examined on skin model under UVB irradiation. This exvivo human organ skin model has already been reported and serves as an appropriate tool for photo-damage elucidation [21].

Topical application of DSOC preparation had protected the epidermis from UVB-mediated cytotoxicity characterized by apoptosis induction (Figure 1) and did not impair cell viability as detected by mitochondrial activity (Figure 2). Furthermore, the increase in mitochondrial activity in irradiated skin pre-treated with DSOC is complementary to the anti-apoptotic effect of this preparation with regards to irradiated samples.

Osmoter $^{\mathrm{TM}}$, one of the components of DSOC preparation, was already reported to dramatically reduce the number of apoptotic cells following exposure to UVB in keratinocyte cultures [11]. Yet, it increased the expression of Fas metabolic pathway leading to "extrinsic apoptosis" Since Fas expression is turned off in skin pathological conditions such as carcinomas, we assume that Fas may contribute to skin anti tumoral protection in damaged keratinocytes [22].Thus, Osmoter ${ }^{\mathrm{TM}}$ might protect apoptosis selectively by enhancing the expression of Fas receptor to limit pathogenic carcinogenic threats and by reducing "intrinsic apoptosis" to protect normal cells.

The decrease in UVB-induced apoptosis following application of DSOC preparation might be also due to the potent strong antioxidant activity of DSOC preparation plant extracts assisting in excess ROS scavenging. Studies have demonstrated that overproduction of reactive oxygen species (ROS) plays a role in the process of apoptosis by triggering multiple signaling pathways involving pro-caspases, cytochrome $\mathrm{C}$, apoptosis-inducing factor (AIF), and apoptotic protease-activating factor-1 (APAF-1) [23]. As reported, extracts of plants included in the DSOC, i.e. Grape seed, Green tea leaf and Olive leaf, inhibit the ROS activity by their poly-phenoles possessing scavenging capacities,

Excess of ROS production might also lead to the ac- 
cumulation of oxidatively modified proteins. Proteasome, the major intracellular proteolytic system implicated in the removal of abnormal and oxidized protein, has a pivotal role in cellular detoxification. Previous studies in human epidermal cells have shown that proteasome activity is decreased during senescence as well as upon UV irradiation [24]. It has been shown that keratinocytes UVA and UVB irradiation led to a decrease in proteasome peptidase activity. 20S sub-unit was found to be very sensitive in its active form to UVA- and UVB-irradiation mainly affecting the peptidylglutamyl-peptide hydrolase activity [24]. In this study, proteasome 20S sub-unitchymotrypsin-like activity and trypsine-like activity indeed decreased in skin organ cultures following exposure to UVB irradiation (Figure 3). Pre-treatment with DSOC preparation abolished this decrease and thus, assisted in maintaining protein turnover, which is essential to preserve cell function. This finding can be related to DSOC preparation neutralizing ROS formation. A study published in 2011 article by Koziel et al. suggests a functional interplay between mitochondrial activity and proteasome activity in skin aging, proposing that both systems can be associated in skin aging [25]. Since DSOC is shown to induce mitochondrial activity, indeed, this correlation has been found between the two functions with regards to UVB photoaging.

One major result of photo-damage is the dehydration of the skin [8]. Aquaporin 3 (AQP3)-membrane-inserted water channel play an important role in the regulation of water permeability [7]. Indeed, pre-treatment with DSOC markedly enhanced AQP3 expression in epidermis (Figure 4).

AQP3 expression has been linked to various skin dis-

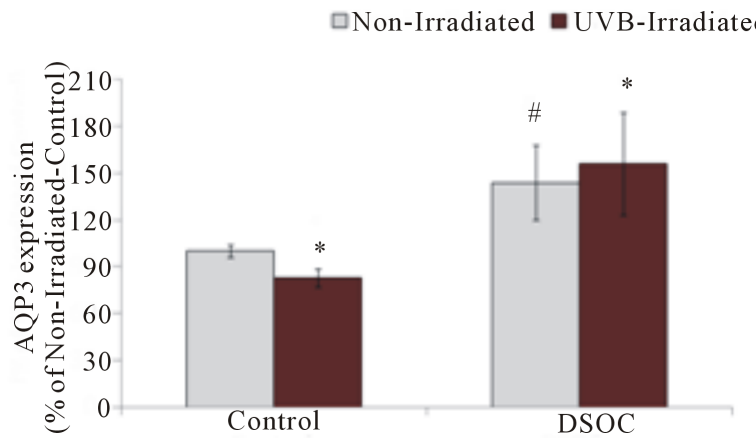

Figure 4. Epidermal AQP3 expression following treatment with DSOC preparation and UVB irradiation. Human skin pieces were applied with DSOC preparation for $24 \mathrm{hrs}$ and UVB irradiated at $250 \mathrm{~mJ} / \mathrm{cm}^{2}$ after the removal of preparation. AQP3 protein expression was evaluated using Dotblot analysis in epidermal extracts $48 \mathrm{hr}$ following irradiation. Data are presented as Mean \pm SEM. ${ }^{*}$ p $<0.05$ Irradiated-DSOC applied vs. Irradiated Control; ${ }^{\#} p=0.05$ Non-Irradiated-DSOC applied vs. Non-Irradiated Control. orders. Based on a study showing the aquaporin elevation after $\mathrm{MgSO}_{4}$ administration in rat [26], it might be speculated that in the case of DS water, the rich content of magnesium salt could also contribute to elevation in AQP3 expression.

Previous studies have demonstrated that UV radiation down-regulates AQP3 expression in cultured skin keratinocytes, leading to a decrease in water permeability also due to the reduced glycerol transport through AQP3 [27].

AQP3 down-regulation can also be due to UV-induced ROS over production via activating the MAPK/ERK (mitogen-activated protein kinases/extracellular signalregulated kinase) pathway as shown in cultured skin keratinocytes [28].

Here, we show that AQP3 protein expression is decreased following UVB induction in human skin organ model while apre-treatment with DSOC preparation has a protective effect against UVB by increasing AQP3 levels (Figure 4).

Based on its beneficial effects on human skin organ culture model in different modes of biological activities, DSOC preparation was formulated into a serum (DSOC Serum) for facial skin and was tested for skin hydration.

Skin hydration is important for its health and functionality. A decrease in skin moisturization level is related to different biological processes and stressors (i.e., aging, UV radiation, autoimmune skin disorders, and harsh environmental climates) and might lead to the impairment in skin barrier function, enhancement in skin roughness, and decrease of skin elasticity and firmness[21,29].

DSOC Serum demonstrates intense moisturizing effects by significantly increase SC hydration level (Figure 5). The improvement in hydration was the higher relatively to subjects regular moisturizer. Furthermore, DSOC serum boosts the effect of hydration together with the regular moisturizer. These results from the clinical study may reflect in addition to moisture at the skin surface level via hygroscopic molecules and emollients, the positive effect at the cellular level of DSOC on AQP3. Additional studies can be performed to elucidate the contribution of other actives in the DSOC Serum to skin.

\section{Conclusion}

In conclusion, we showed that a selection of plant extracts together with DS water extract (DSOC preparation) can significantly assist the skin in coping with UVB rays stress via anti-apoptotic properties and by maintaining normal proteasome activity and AQP3 expression. For the first time, it was demonstrated that concentrated DS water combined with plant extracts affect directly on proteasome activity and AQP3 expression. Hence, DSOC 


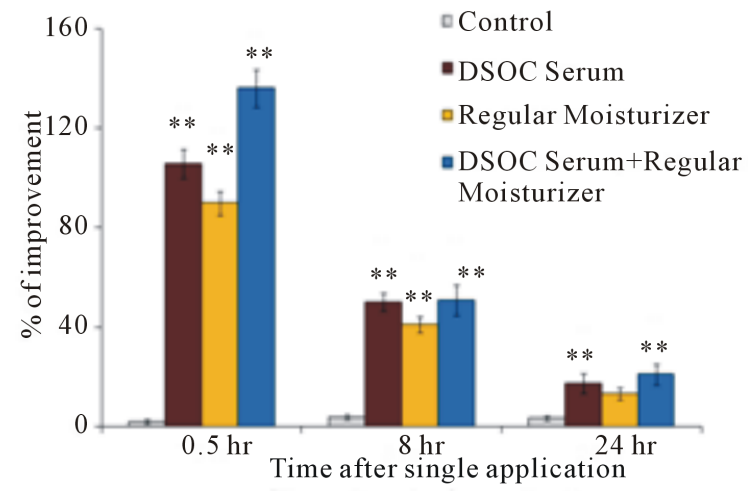

Figure 5. Skin hydration between control forearm and tested hand forearm at different time points following application of DSOC Serum compared to other treatments. Skin hydration in the flexor aspect of the forearm was measured in 35 female volunteers using a corneometer as described at the methods section. The measurements were performed in defined periods after a single application in $\mathbf{4}$ different application areas for DSOC Serum, Regular Moisturizer of the subjects, DSOC Serum plus the Regular Moisturizer and control (non-applied) area. The relative improvement between each treatment and control area was calculated. Data are presented as Mean \pm SEM for delta (\%), i.e. percentage moisture change caused by the application, with reference to the starting value (before application). ${ }^{* *} \mathrm{p}<\mathbf{0 . 0 1}$.

protects skin from photo-damage simultaneously on different cellular functions.

DSOC preparation implemented in a cosmetic formula (DSOC Serum) was shown in a clinical study to increase significantly skin moisture and to also boost the moisturizing capacity of regular moisturizer and is thus, expected to improve facial skin appearance. Further studies are needed to complete the understanding of DS minerals on skin moisture. The impact of UVA is needed to fully examine the protective effect of DSOC of aquaporin and proteasomal function against photo-damage.

\section{Acknowledgements}

We would like to thank AHAVA formulation team that took part in the development of DSOC preparations; Marina Rekhter, Juilia Gorovetz and Nataly Miller.

We also would like to thank Dr. David ZN Frankel from Atidim, Medicla center, Tel-Aviv for the supplementation of surplus human skin from aesthetic surgeries.

\section{REFERENCES}

[1] Y. Miyachi and O. Ishikawa, "Dermal Connective Tissue Metabolism in Photoageing,” Australasian Journal of Dermatology, Vol. 39, No. 1, 1998, pp. 19-23. http://dx.doi.org/10.1111/j.1440-0960.1998.tb01235.x
[2] S. Pillai, C. Oresajo and J. Hayward, "Ultraviolet Radiation and Skin Aging: Roles of Reactive Oxygen Species, Inflammation and Protease Activation, and Strategies for Prevention of Inflammation-Induced Matrix Degradation -A Review," International Journal of Cosmetic Science, Vol. 27, No. 1, 2005, pp. 17-34. http://dx.doi.org/10.1111/j.1467-2494.2004.00241.x

[3] T. M. Runger, "Role of UVA in the Pathogenesis of Melanoma and Non-Melanoma Skin Cancer. A Short Review," Photodermatology, Photoimmunology \& Photomedicine, Vol. 15, No. 6, 1999, pp. 212-216. http://dx.doi.org/10.1111/j.1600-0781.1999.tb00090.x

[4] M. Ichihashi, M. Ueda, A. Budiyanto, T. Bito, M. Oka, M. Fukunaga, K. Tsuru and T. Horikawa, "UV-Induced Skin Damage,” Toxicology, Vol. 189, No. 1-2, 2003, pp. 21-39. http://dx.doi.org/10.1016/S0300-483X(03)00150-1

[5] R. Kohen and A. Nyska, "Oxidation of Biological Systems: Oxidative Stress Phenomena, Antioxidants, Redox Reactions, and Methods for Their Quantification," Toxicologic Pathology, Vol. 30, No. 6, 2002, pp. 620-650. http://dx.doi.org/10.1080/01926230290166724

[6] S. Verdier-Sevrain and F. Bonte, "Skin Hydration: A Review on Its Molecular Mechanisms," Journal of Cosmetic Dermatology, Vol. 6, No. 2, 2007, pp. 75-82. http://dx.doi.org/10.1111/j.1473-2165.2007.00300.x

[7] A. Watkinson, C. Harding, A. Moore and P. Coan, "Water Modulation of Stratum Corneum Chymotryptic Enzyme Activity and Desquamation," Archives of Dermatological Research, Vol. 293, No. 9, 2001, pp. 470-476. http://dx.doi.org/10.1007/s004030100257

[8] S. J. Shan, T. Xiao, J. Chen, S. L. Geng, C. P. Li, X. Xu, Y. Hong, C. Ji, Y. Guo, H. Wei, W. Liu, D. Li and H. D. Chen, "Kanglaite Attenuates UVB-Induced Down-Regulation of Aquaporin-3 in Cultured Human Skin Keratinocytes,” International Journal of Molecular Medicine, Vol. 29, 2011, pp. 625-629.

[9] S. Halevy and S. Sukenik, "Different Modalities of Spa Therapy for Skin Diseases at the Dead Sea Area," Archives of Dermatology, Vol. 134, No. 11, 1998, pp. 14161420. http://dx.doi.org/10.1001/archderm.134.11.1416

[10] S. W. Moses, M. David, E. Goldhammer, A. Tal and S. Sukenik, "The Dead Sea, a Unique Natural Health Resort," Israel Medical Association Journal, Vol. 8, 2006, pp. 483-488.

[11] Y. Soroka, Z. Ma’or, Y. Leshem, L. Verochovsky, R. Neuman, F. M. Bregegere and Y. Milner, “Aged Keratinocyte Phenotyping: Morphology, Biochemical Markers and Effects of Dead Sea Minerals,” Experimental Gerontology, Vol. 43, No. 10, 2008, pp. 947-957. http://dx.doi.org/10.1016/j.exger.2008.08.003

[12] Z. Ma'or, S. Yehuda and W. Voss, "Skin Smoothing Effects of Dead Sea Minerals: Comparative Profilometric Evaluation of Skin Surface," International Journal of Cosmetic Science, Vol. 19, No. 3, 1997, pp. 105-110. http://dx.doi.org/10.1111/j.1467-2494.1997.tb00173.x

[13] X. Wang, F. Bregegere, Y. Soroka, A. Kayat, G. Redziniak and Y. Milner, "Enhancement of Fas-Mediated Apoptosis in Ageing Human Keratinocytes,” Mechanisms of 
Ageing and Development, Vol. 125, No. 3, 2004, pp. 237249. http://dx.doi.org/10.1016/j.mad.2003.12.007

[14] Y. Matsumura and H. N. Ananthaswamy, "Toxic Effects of Ultraviolet Radiation on the Skin,” Toxicology and Applied Pharmacology, Vol. 195, No. 3, 2004, pp. 298308. http://dx.doi.org/10.1016/j.taap.2003.08.019

[15] M. Iwata, T. Takebayashi, H. Ohta, R. E. Alcalde, Y. Itano and T. Matsumura, "Zinc Accumulation and Metallothionein Gene Expression in the Proliferating Epidermis during Wound Healing in Mouse Skin," Histochemistry and Cell Biology, Vol. 112, No. 4, 1999, pp. 283-290. http://dx.doi.org/10.1007/s004180050449

[16] Z. Y. Wang, R. Agarwal, D. R. Bickers and H. Mukhtar, "Protection against Ultraviolet B Radiation-Induced Photocarcinogenesis in Hairless Mice by Green Tea Polyphenols,” Carcinogenesis, Vol. 12, No. 8, 1991, pp. 15271530. http://dx.doi.org/10.1093/carcin/12.8.1527

[17] D. Bagchi, M. Bagchi, S. J. Stohs, D. K. Das, S. D. Ray, C. A. Kuszynski, S. S. Joshi and H. G. Pruess, "Free Radicals and Grape Seed Proanthocyanidin Extract: Importance in Human Health and Disease Prevention," Toxicology, Vol. 148, No. 2-3, 2000, pp. 187-197. http://dx.doi.org/10.1016/S0300-483X(00)00210-9

[18] S. K. Mantena and S. K. Katiyar, "Grape Seed Proanthocyanidins Inhibit UV-Radiation-Induced Oxidative Stress and Activation of MAPK and NF-KappaB Signaling in Human Epidermal Keratinocytes,” Free Radical Biology and Medicine, Vol. 40, No. 9, 2006, pp. 1603-1614. http://dx.doi.org/10.1016/j.freeradbiomed.2005.12.032

[19] M. A. Carluccio, L. Siculella, M. A. Ancora, M. Massaro, E. Scoditti, C. Storelli, F. Visioli, A. Distante and R. De Caterina, "Olive Oil and Red Wine Antioxidant Polyphenols Inhibit Endothelial Activation: Antiatherogenic Properties of Mediterranean Diet Phytochemicals,” Arteriosclerosis, Thrombosis, and Vascular Biology, Vol. 23, 2003, pp. 622-629. http://dx.doi.org/10.1161/01.ATV.0000062884.69432.A0

[20] Y. Kimura and M. Sumiyoshi, "Olive Leaf Extract and Its Main Component Oleuropein Prevent Chronic Ultraviolet B Radiation-Induced Skin Damage and Carcinogenesis in Hairless Mice,” Journal of Nutrition, Vol. 139, No. 11, 2009, pp. 2079-2086. http://dx.doi.org/10.3945/jn.109.104992

[21] M. Portugal-Cohen, Y. Soroka, M. Frusic-Zlotkin, L. Verkhovsky, F. M. Bregegere, R. Neuman, R. Kohen and Y. Milner, "Skin Organ Culture as a Model to Study Oxidative Stress, Inflammation and Structural Alterations Associated with UVB-Induced Photodamage,” Experimental
Dermatology, Vol. 20, No. 9, 2011, pp. 749-755. http://dx.doi.org/10.1111/j.1600-0625.2011.01317.x

[22] E. Filipowicz, P. Adegboyega, R. L. Sanchez and Z. Gatalica, "Expression of CD95 (Fas) in Sun-Exposed Human Skin and Cutaneous Carcinomas,” Cancer, Vol. 94, No. 3, 2002, pp. 814-819. http://dx.doi.org/10.1002/cncr.10277

[23] K. Kannan and S. K. Jain, "Oxidative Stress and Apoptosis,” Pathophysiology, Vol. 7, No. 3, 2000, pp. 153-163. http://dx.doi.org/10.1016/S0928-4680(00)00053-5

[24] A. L. Bulteau, M. Moreau, C. Nizard and B. Friguet, "Proteasome and Photoaging: The Effects of UV Irradiation,” Annals of the New York Academy of Sciences, Vol. 1100, 2007, pp. 280-290.

http://dx.doi.org/10.1196/annals.1395.029

[25] R. Koziel, R. Greussing, A. B. Maier, L. Declercq and P. Jansen-Durr, "Functional Interplay between Mitochondrial and Proteasome Activity in Skin Aging,” Journal of Investigative Dermatology, Vol. 131, 2011, pp. 594-603. http://dx.doi.org/10.1038/jid.2010.383

[26] N. Ikarashi, T. Ushiki, T. Mochizuki, T. Toda, T. Kudo, K. Baba, M. Ishii, K. Ito, W. Ochiai and K. Sugiyama, "Effects of Magnesium Sulphate Administration on Aquaporin 3 in Rat Gastrointestinal Tract," Biological and Pharmaceutical Bulletin, Vol. 34, No. 2, 2011, pp. 238242. http://dx.doi.org/10.1248/bpb.34.238

[27] C. Cao, S. Wan, Q. Jiang, A. Amaral, S. Lu, G. Hu, Z. Bi, N. Kouttab, W. Chu and Y. Wan, "All-Trans Retinoic Acid Attenuates Ultraviolet Radiation-Induced Down-Regulation of Aquaporin-3 and Water Permeability in Human Keratinocytes,” Journal of Cellular Physiology, Vol. 215, No. 2, 2008, pp. 506-516. http://dx.doi.org/10.1002/jcp.21336

[28] S. Kang, J. H. Chung, J. H. Lee, G. J. Fisher, Y. S. Wan, E. A. Duell and J. J. Voorhees, "Topical N-Acetyl Cysteine and Genistein Prevent Ultraviolet-Light-Induced Signaling that Leads to Photoaging in Human Skin in Vivo," Journal of Investigative Dermatology, Vol. 120, 2003, pp. 835-841. http://dx.doi.org/10.1046/j.1523-1747.2003.12122.x

[29] E. Wineman, M. Portugal-Cohen, Y. Soroka, D. Cohen, G. Schlippe, W. Voss, S. Brenner, Y. Milner, N. Hai and Z. Ma'or, "Photo-Damage Protective Effect of Two Facial Products, Containing a Unique Complex of Dead Sea Minerals and Himalayan Actives," Journal of Cosmetic Dermatology, Vol. 11, No. 3, 2012, pp. 183-192. http://dx.doi.org/10.1111/j.1473-2165.2012.00625.x 